Rev. Elev. Méd. Vét. Poys trop., 1966, 19, 2 (149-162)

\title{
Helminthes des zébus adultes de la région de Maroua (Nord-Cameroun)
}

\author{
par M. GRABER, R. FERNAGUT ef O. OUMATIE
}

\begin{abstract}
RÉSUMÉ
Une enquête menée à l'abattorr de Maroua (Nord-Cameroun) et portant sur 409 animaux de boucherı a permis de mettre en évidence sur le bétall autochtone 30 espèces différentes d'Helminthes dont les mieux représentées sont : Dicrocoelium hospes $(61,3$ p. 100$)$, Fasciola gigantica (31,3 p. 100), divers Poramphistomidae de la panse (52 p. 100), cinq Cormyerius (35,3 p. 100), Schistosoma bovis (28,8 p. 100), Cysticercus bovis (21 p. 100), de nombreux Trichostrongylidae (Haemoncus contortus, 52 p. 100 ; Cooperio pectinata et Cooperia punctata, 21 p. 100) et des Filaires (Artionema labiato-papillosa, 29,5 p. 100 ; Onchocerca armillato, 29,5 p. 100 ; Onchocerca gulturosa, 43,2 p. 100). Le parasitisme par Oesophagostornum radiotum, Bunostamum phlebofomum et les grands Cestodes de l'intestin grêle paraît assez madéré.

Ces Helminthes sont associés entre eux dans la quasi-lotalité des cos ef les associations sont graves, car elles mettent en jeu souvent six, huit et même neuf espèces dont cerlaines sont très pathogènes.

Les auteurs comparent le parasıtisme du zébu adulte nord-Camerounais au parasitisme global des zébus du Tchad ef de R. C. A. (Bouar). Ils notent un accroissement sensible du taux d'infestation des animaux des zones sahéliennes (isohyète $300-500 \mathrm{~mm}$ ) lorsqu'ils descendent pour abattage vers les régions plus humides (isohyète $800-900 \mathrm{~mm}$ ).
\end{abstract}

\section{GÉNÉRALITÉS}

10 Le Pays.

La région intéressée représente le versant camerounals de la cuvette tchadienne. Comprise entre le $10^{\mathrm{e}}$ et le $13^{\mathrm{e}}$ de latitude Nord et le $14^{\mathrm{e}}$ et le $16^{\text {e }}$ de longifude est, elle est limitée

- au Sud par la ligne de partage des eaux de la Bénaué

- au Nord par le lac Tchad

- à l'Ouest par le massif montagneux du Mandaro

- à l'Est par le fleuve Logone.

II s'agit d'une vaste plaine drainée tant bien que mal par le Logone et le Charı qui servent de frontière. A l'intérieur, les cours d'eau ou «mayos» dont la crue est maximum de juillet à actobre descendent des monts de Mandara, traversent la plaine alluviale et vont se perdre dans l'immense marécage que constituent les «Yaérés $»$ de Pouss et de Guirvidic. Au cours de leur trajet, ils remplissent de nombreuses dépressions qui constituent autant de mares plus ou moins permanentes.

Le Nord-Cameroun, surtout dans les préfectures de Mayo-Dana et du Diamaré d'où esł orıgınare le tétail dont il va être question dans cet article est donc un pays bas, relativement humide, riche de collections d'eau de toute nature. Cette situation est partıculièrement favorable à la pullulation de certains vecteursmoustiques et mollusques - qui ont besoin pour survivre d'un milieu aquatique ou semi-aquati- 


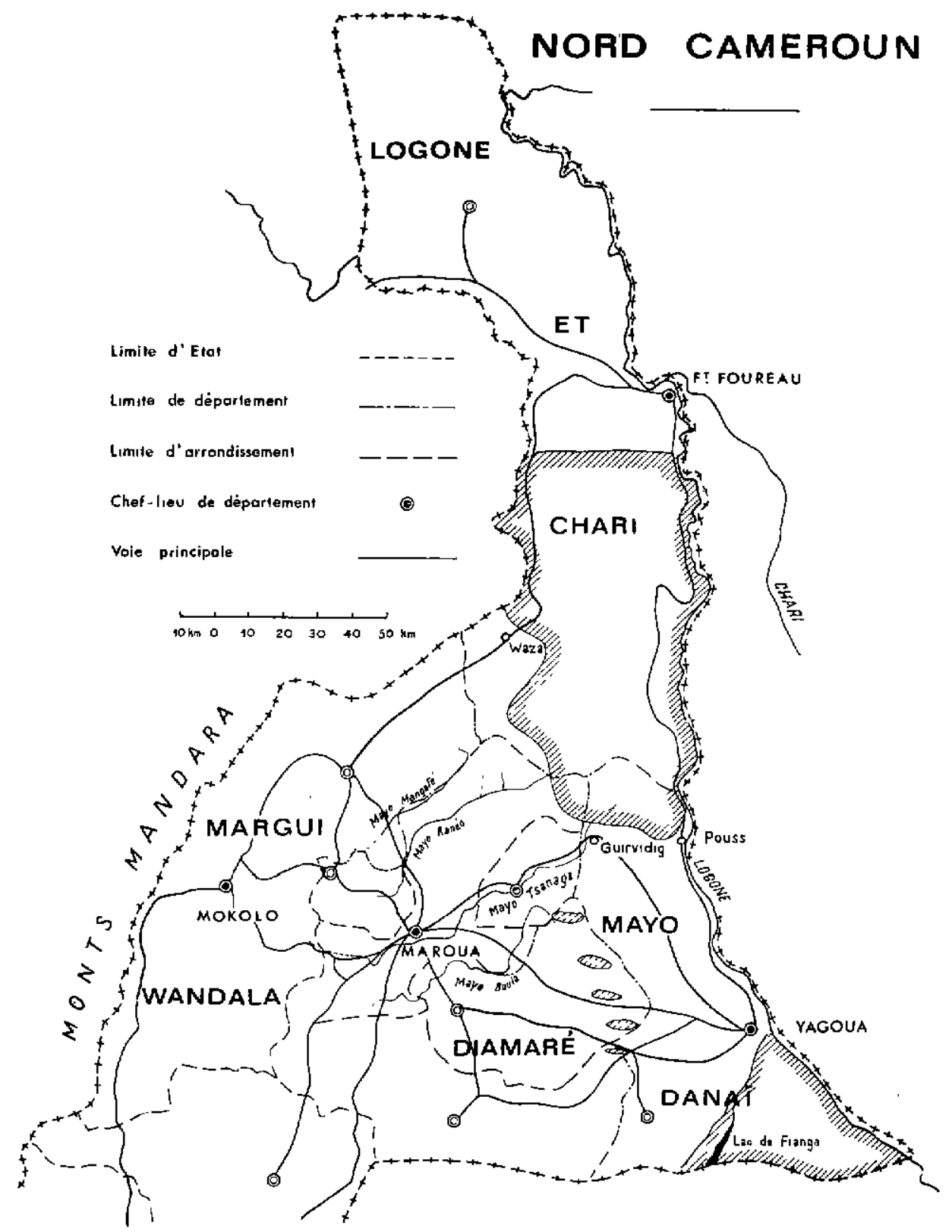


que permanent. La plaine est formée de sédiments d'origine quaternaire: argiles compactes ou à retrait, alluvions anciennes ou récentes plus fertiles.

\section{Le Climat.}

II est de type soudano-sahélien avec deux saisons bien tranchées: la saison sèche (d'octobre au 15 avril) et la saison des pluies (d'avril à la fin septembre).

La moyenne des précipitations mensuelles (en mm) relevées de 1948 à 1957 est la suivante :

$\begin{array}{llll}\text { Janvier : } & 0 & \text { Juillet } & : 184,5 \\ \text { Févirer : } 0,3 & \text { Aoôt }: 236,5 \\ \text { Mars : } 2,7 & \text { Septembre }: 151 \\ \text { Avril }: 10,4 & \text { Octobre : } 29,7 \\ \text { Mai }: 76,9 & \text { Novembre : } 0 \\ \text { Juin : } 97,7 & \text { Décembre : } 0\end{array}$

Les premières pluies apparaissent en avril; elles vont en s'intensifiant peu à peu en maijuin. Du 20 juin au 10 juillet, s'instalie une courte saison sèche. Le maximum est atteint au cours des deux dernières décades d'août.

Il est bon de noter que la moyenne pluviométrique annuelle décroît en Sud-Ovest NordEst : suivant l'axe des vents dominants, cette diminution est de l'ordre de $100 \mathrm{~mm}$.

Le degré hygrométrique accuse de forts écarts, le minimum se situant autour de 8 p. 100 en février et le maximum (plus de 80 p. 100) en août-septembre. Quant à la température, l'amplitude absolve est d'environ $35^{\circ} \mathrm{C}$ (minimum $10^{\circ} \mathrm{C}$; maximum $45^{\circ} \mathrm{C}$ ). Comme dans toutes ces régions, la température maxımum moyenne s'observe en mars-avril; elle diminue ensuite, passe par un minimum en août, remonte jusqu'en novembre pour revenir au minimum en janvier.

La température minimale moyenne subit des fluctuations analogues.

\section{3o Le Bétail.}

Le versant camerounass de la cuvette tchadienne est peuplée d'environ 550.000 zébus, 500.000 ovins ef d'un million de caprins.

Ce bétail est, dans l'ensemble, de qualité médiocre et assez peu exploitable pour la boucherie. Pour explıquer cet état de choses, plusieurs raisons peuvent être invoquées, les unes d'ordre alimentaire (concurrence des cultures ef des herbages, ce qui entraîne une diminution des surfaces utilisées par le bétail, donc un déficit alimentaire), les autres d'ordre pathologique. A cet égard, la lutie contre les maladies contagieuses est très avancée et de grands progrès ont été réalisés. II n'en est malheureusement pas de même des maladies parasıtaires qui, par leur. incidence économique, représentent le plus gros problème du Nord-Cameroun. Les trypanosomiases restent graves malgré une lutte soutenue. Quant aux Heiminthiases, elles n'ont encore fait l'objet d'aucune étude systématique.

Le but du 'présent travail est de tenter de combler cette lacune. Pour l'instant, il ne sera question que des parasites des animaux de boucherie, les Helminthiases des jeunes devant être traitées ultérieurement.

\section{MATÉRIEL ET MÉTHODE}

Les enquêtes ont commencé en 1963 et ont été terminées l'année suivante. Les Helminthes ont été recueillis à l'autopsie de zébus adultes sacrlfiés à l'abattoir de Maroua.

La région du Nord-Cameroun étant une zone de transhumance, plusieurs sondages ont été effectués :

- en saison des pluies (coût-septembre 1963 et 1964) où, du fait de la coupure des routes, il est absolument certain que le bétail examıné est né et a été élevé dans la région ;

- en saison sèche (janvier-février 1964) : il s'agit alors en grande majorité d'animaux venu du Tchad et achetés à des Nomades de passage ou sur les marchés de la zone sahélienne.

L'étude du parasitısme de ces deux populations bovines permet d'utiles comparaisons.

Au total, 409 zébus adultes ont été soumis à un examen minutieux dont :

140 en août-septembre 1963

115 en janvier-février 1964

154 en août-septembre 1964

Les parasites ont été récoltés sur place selon tes techniques habituelles (Euzéby, 1958), formolés et expédiés au Laboratoire de Farcha (Fort-Lamy) où 'Ils ont été déterminés, pesés ou comptés. 


\section{HELMINTHES EN CAUSE LEUR LOCALISATION}

\section{A. - Parasites stomacaux}

\section{Panse.}

10 Paramphistomum microbothrium (Fischoeder, 1901).

Ce Trématode est largement répandu en Afrique (Afrique du' Sud, Kenya, Angola, Congo, Afrique de l'Ouest). Ce n'est qu'en 1937 (Nâsmark) que Poromphistomum cervi reconnu dans diverses régions d'Afrique a été rapporté sûrement à Paramphistomum microbothrium. Ce parasite est très fréquent dons la panse des animaux domestiques et sauvages de la cuvette tchadienne, beaucoup moins en R. C. A. (Bouar).

20 Colicophoron calicophorum (Fischoeder, 1901),

3o Gigantocotyle symmeri (Näsmark, 1937) a été redécrit par Nâsmark à partir de cinq exemplaires et d'une série de coupes sagittales déposés dans la collection de Looss qui l'avait nommé Amphistomum gigantocotyle. Le matériel original avait été récolté par Symmer à l'abattoir du Caire sur des animaux venus du Soudan Nilotique.

Le même parasite a été retrouvé dans la région du Mayo-Kebbi (Tchad) voisine du Nord-Cameroun.

40 Stephanopharynx compoctus (Fischoeder, 1901). S. compactus existe dans différents territoires africains (Rhodésie, Tchad).

50 Bothriophoron bothriophoron (M. Braun, 1892). B. bothriophoron a été décrit par Braün à partir de quatre exemplaires recueillis dans la panse d'un zébu à Tananarive (Zool. museum de Königsberg).

Par la suite, ce Trématode a été mis en synonymie avec Paromphistomum cervi Gretillat (1958) ressuscite le genre et l'espèce, toujours à partir de Trématodes originaires de la grande île (lac Alaotra, région de Majunga...). Dinnik (Gretillat, 1958) le signale da Nairobi dans le rumen d'un mouton et d'une chèvre.

En 1960, B. bothriophoron est de nouveau mis en évidence, mais en Afrique centrale chez un Bos taurus (Kouri) abattu à Fort-Lamy.

L'espèce est très abondante chez les zébus de la région de Maroua. La cuvette tchadienne est donc le troisième point d'Afrique à héberger ce parasite.

$6^{\circ}$ Carmyerius spatiosus (Brandes, 1898).

Très largement réparti en Afrique au Sud du Sahora, ce Carmyerius est commun tant chez les ruminants domestiques que chez les Artiodactyles sauvages (Graber et coil., 1964).

70 Carmyerius groberı (Gretillat, 1960)

déborde les limites du Tchad, puisqu'il a été revu au Congo (Gretillat, 1964) au Cameroun et en R. C. A. (Bouar).

80 Cormyerius papillatus (Gretillat, 1962).

9o Carmyerius parvipopillatus (Gretillat, 1962). Considérés au début comme des parasites d'Artiodactyles sauvages, ces deux Carmyerius sant susceptibles d'infester également les ruminants domestiques. Pour l'instont, $C$. papillatus, comme c. parvipapillatus, sont des Trématodes de la cuvette tchadienne et de son rebord.

10. Carmyerius gregorius (Looss, 1896) a été vu à de nombreuses reprises, dans la panse des ruminants domestiques et sauvages d'Afrique noire. II est rare au Tchad.

Au Nord-Cameroun, les Trématodes de la panse comprennent au moins dix espèces différentes, la plupart du temps associées par 3 ou 4, quelquefois plus, ce qui explique les difficultés considérables que soulève la mise à jour de telles collections.

Il est bon de souligner également la richesse parasitaire de la panse du zébu camerounais, richesse qui dépasse de loin celle du zébu tchadien et du zébu de R. C. A. (Bouar) où Paramphistomum microbothrium est l'espèce dominante dans le premier cas et Cotylophoron cotylophorum dans le second.

\section{Caillefte.}

10 Hoemoncus contortus (Rudolph1, 1803)

20 Haemoncus placel (Place, 1893).

Ce sont des Trichostrongylidés fréquents dans toute l'Afrique centnale.

\section{B. - Parasites de l'intestin grêle}

\section{Cestodes.}

10 Moniezio exponsa (Rudolphi, 1810). 
20 Moniezıa beneden (Moniez, 1879).

$3^{\circ}$ Thysoniezia ovilla (Rivolta, 1878).

Ces Anoplocephalidae sont des espèces classiques, particulièrement abondantes dans les zones chaudes et sèches d'Afrique centrale.

\section{Nématodes.}

10 Bunostomum phlebotomum (Roilliet, 1900).

$2^{\circ}$ Cooperio punctata (Von Linstow, 1907).

$3^{\circ}$ Cooperia pectinato (Ramsom, 1907).

Là encore, il s'agit d'espèces très souvent rencontrées en 'Afrıque noire.

\section{C. - Parasites du gros intestin ef du cæcum}

10 Oesophagostomum (Bosicolo) rodiatum (Rudolphi, 1803).

20 Buckleyuris globuloso (Von Linstow, 1901).

\section{D. - Parasites de l'appareil vaśculaire}

\section{Trématodes.}

10 Schistosoma bovis (Sonsino, 1876).

20 Schistosomio mottheer (Veglia et Le Roux, 1927).

Ces deux Schistosomes existent aussi dans les régions voisines du Tchad (Logone, Mayo-Kebbi, Chari-Baguirmi et'Lac), chez les ruminants domestiques comme chez les ruminants sauvages. Par contre, ils paraissent rarissime en R. C. A.

Schistosoma curossoni n'a pu jusqu'à maintenant être mis en évidence : le Nord-Cameroun semble, jusqu'à plus ample informé, se rattacher plutôt au système de l'Est africain riche en Schistosoma bovis (région Nilotıque) qu'au système de l'Ouest africain où $S$. curassoni tient une place importante.

La schistosomiase à S. bovis se traduit par des signes d'entérite avec souvent hémorragie au niveau de l'intestin et émission de selles sanglantes qui peuvent en imposer pour de la Coccidiose. Cette bilharziose se differentie donc nettement de la bilharziose à S. curassoni qui est plutôt de type hepatico-pulmonaire (Gretillat et Picart, 1964).

\section{Nématodes.}

1. Onchocerca ormillata (Railliet et Henry, 1909) est une filaire banale de la crosse de l'aorte.
L'existence de ce parasite a été isignalée en République démocratique du Congo (Hérin et Coll., 1955 ; Fain et Coll., 1955), au Ghana (Chodnik, 1957 et 1958) et au Soudan (Abdel Malek, 1958) où le pourcentage d'animaux infestés dépasse 95 p. 100 dans les deux provinces du Nil Blanc ef du Kordofan. Au Tchad, le taux d'infestation des animaux adultes est légèrement supérieur à 40 p. 100 (Graber, 1965).

\section{E. - Parasites du foie}

\section{Canaux biliaires et vésicule.}

10 Fasciola gigantica (Cobbold, 1855).

20 Dicrocoelium hospes (Looss, 1907).

Ce dicrocoelidae vit dans les canaux biliaires et la vésicule du boeuf. Quelques exemplaires ont également été retrouvés dans le duodénum (10 p. 100 environ). II existe actuellement de par le monde deux espèces de Dicrocoelium parasites des canaux biliaires des animaux domestiques :

- D. lanceolatum (Rudolphi, 1803) en Europe, en Amérique et en Russie.

- D. hospes, espèce décrite pour la première fois par Looss en 1907 chez un bøuf du Soudan Nilotique, revue à plusieurs reprises au Ghana, au Nigeria et plus récemment au Tchad, en R. C. A. et au Congo (Graber et Oumatié, 1964 ; Graber, 1965).

Divers auteurs ont signalé en Afrique noire la présence de Dicrocoelium lanceolatum. If s'agissait vraisemblablement ou de Trématodes récoltés dans le foie d'animaux importés d'Europe ou de confusion dans la détermination du parasite. Dollfus (1965), se basant sur une longue expérience personnelle pense que la «petite Douve » américano-européenne de type D. Ianceodolatum ne vit pas en Afrique.

Dicrocoelum hospes, « la petite douve africaine $»$, est en Afrique centrale la seule espèce connue au-dessous du 11e parallèle, tant chez le bceuf que chez le mouton. Les caractères morphologiques comparés d'exemplaires de $D$. Ionceolotum et de $D$. hospes ne laissent subsister aucun doute quant à l'identité réelle du second parasite.

\section{Tissu hépatique.}

10 Echinococcus polymorphus (Diesing). 
F. - Parasites des muscles et des ligaments

\section{Muscles.}

10 Cysticercus bovis (Cobbold, 1866).

\section{Ligament cervical.}

$2^{\circ}$ Onchocerca gutturosa (Neumann, 1910).

La présence de ce parasite a été notée en République démocratique du Congo (Fain ef Coll., 1955) en R. C. A. (Graber, 1961) et du Tchad (Graber, 1965).

\section{G. - Parasites de la cavité péritonéale}

10 Artionema labiato-papillosa (Perroncito, 1882).

\section{POURCENTAGES 'D'INFESTATION - VARIA- TION DU PARASITISME EN FONCTION DE LA SAISON ET DE L'ORIGINE DES ANI- MAUX SACRIFIÉS}

\section{A. - Taux d'infestation (Tableau I)}

\section{Commentorres}

a) Trente espèces parasites différentes ont été observées chez le zébu adulte de la région de Marova.

b) Ce qui frappe de prime abord, c'est le pourcentage élevé d'animaux atteints. La comparasson entre les pourcentages relevés dans les territoires voisins sur des animaux de même âge donne les résultats suivants (Tableau II) :

Globalement,les taux d'infestation des zébus du Nord-Cameroun sont intermédiaires entre ceux (élevés) de Bouar et ceux (relativement faibles) du Tchad, en ce qui concerne D. hospes, F. gigantica, les Parmphistomidae de la panse, C. bovis, $B$. phlebotomum et Hoemoncus contortus.

Ils dépassent nettement les pourcentages des pays voisins en matière de Gastrothylacidae, S. bovis, Cooperia et diverses Filaires (A. labiatopapillosa et 0 . gutturosa).

Seuls, les Anoplocephalidae de l'intestin sont beaucoup moins bien représentés qu'ils ne le sont au Tchad.

c) Au Nord-Cameroun domine donc un parasitisme à base Te prématodes : Fasciola gigon- tica $(X 2 *)$, Dicrocoelium hospes (X 45), Paramphistomidae $(X 2)$ et Gastrothylacidae $(X 4)$ de la panse, Schistosomes $(=)$.

— L'incidence du Téniasis est limité (trois fois moins).

- L'importance des Nématodes intestinaux (squf pour Cooperia et Hoemoncus) est assez faıble.

- Les Filarioses, sans jouer un rôle essentiel, sont fort nombreuses.

d) «Grosso modo », le schéma général tracé pour les bóvins du Tchad se retrouve au Cameroun, mais le parasitisme va en s'amplifiant : les espèces, sans être beaucoup plus nombreuses, sont plus largement répandues et les infestations

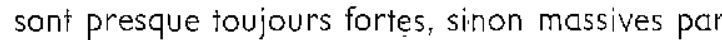
animal, par exemple, 900 grammes de Carmyerius ef 500 grammes de Thysaniezia ovilla).

B. - Variations du parasitisme en fonction de la saison et de l'origine des animaux autopsiés (Tableau III)

\section{Commentaires}

Le parasitisme global, tel qu'ıl a été envisagé dans le paragraphe précédent subit des variations quitiennent:

a) à la saison : le fait est particulièrement probant pour Haemoncus contortus qui disparaît presque complètement en saison sèche, mais touche plus de 50 p. 100 des animaux en saison des pluies :

b) à l'origine des animaux.

Comme il a été dit plus haut, les zébus abattus en janvier-février viennent pour 80 p. 100 environ du Tchad. Ils descendent des marchés du Nord par petites étapes et séjournent quelque temps au Mayo-Kebbi ou au Nord-Cameroun avant d'être tués à Maroua. Pendant leurs déplacements, ils auront toutes chances - comme c'est à peu près la règle pour le bétail africain en cours de commercialisation - de s'infester largement par Paramphistomum, Carmyerius, Schistosomo ou Cysticercus, ce qui explique pourquoi les pourcentages d'infestation observés à Maroua sont bien plus élevés que dans les zones

* Par rapport aux parasites semblables des animaux du Tchad. 
TABLEĀJ NOI

Taux global a'infestation (409 autopsies)

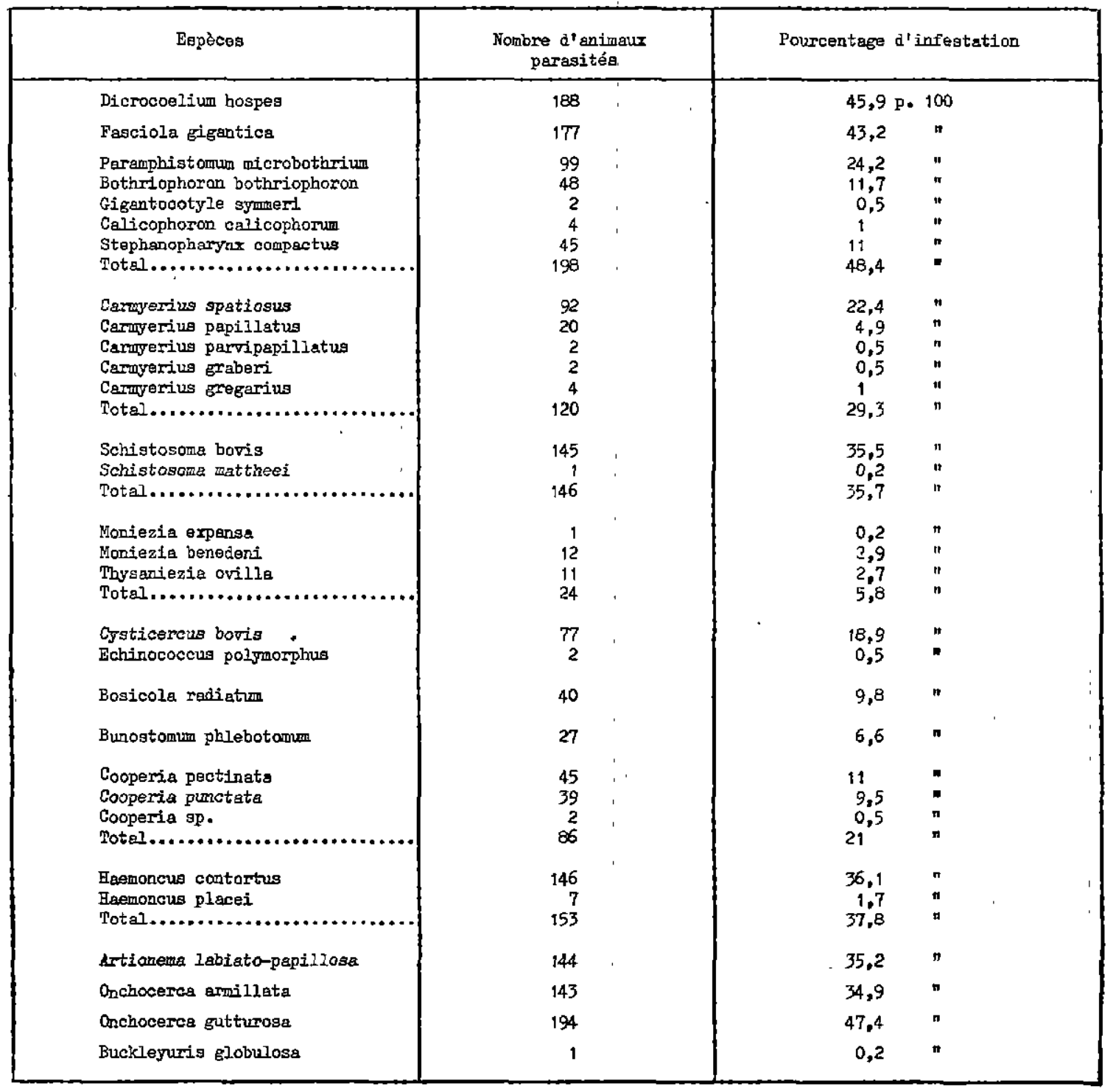


TAELEAD NOII

Taur global d'infestation des zébus adultes du Tchad*, de R.C.A.** et du Cameroun

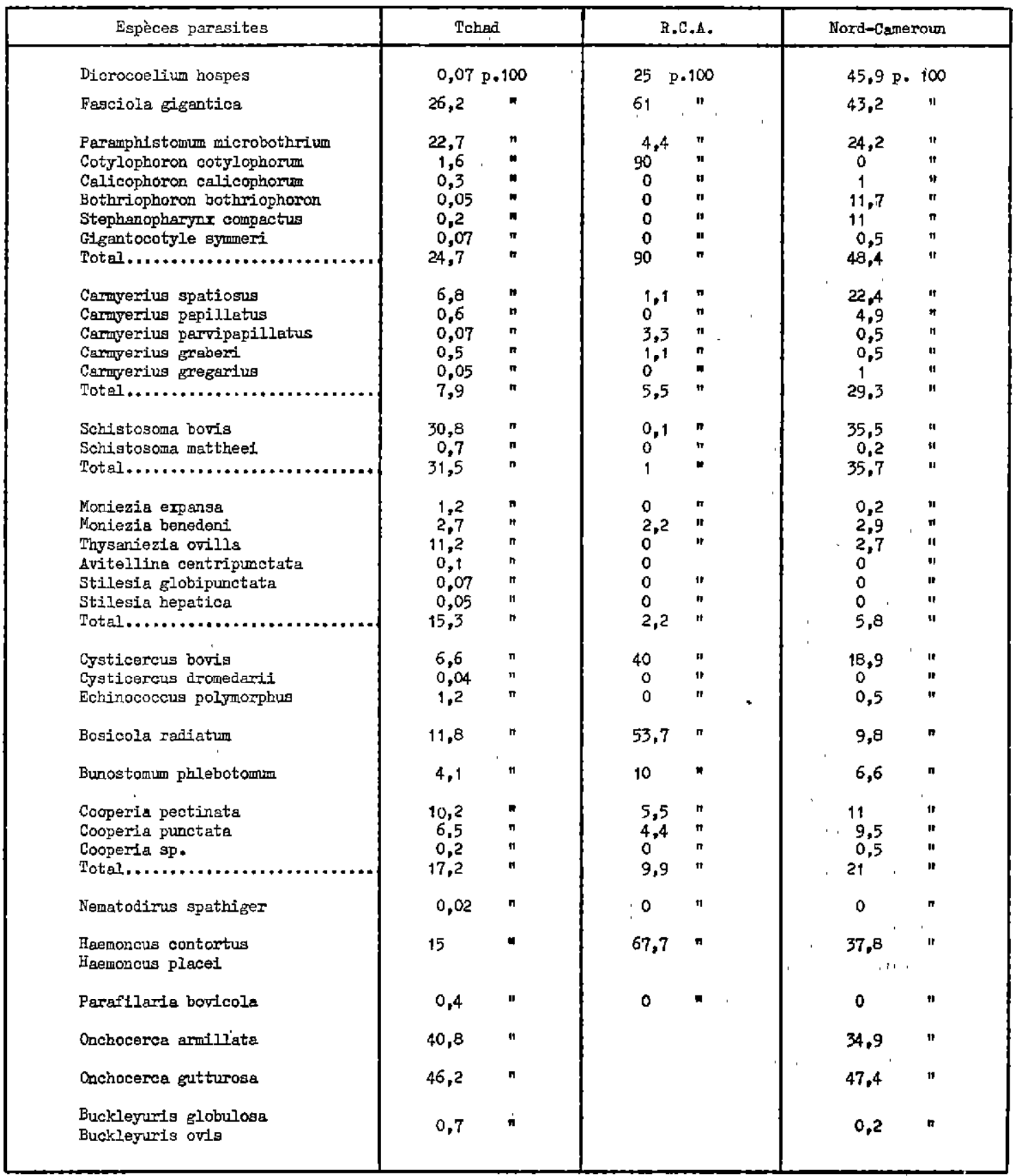


TABLEAU No III

Paregitisme des zébus aduites du nord-Cemeroum en salson secche et en saison des pluies.

\begin{tabular}{|c|c|c|c|c|c|c|}
\hline \multirow[b]{2}{*}{ Espèces en cause } & \multicolumn{3}{|c|}{ Salson sèche=115 animaux } & \multicolumn{3}{|c|}{ Saison des pluies=294 animaux } \\
\hline & $\begin{array}{c}\text { Nombre } \\
\text { d'animaux } \\
\text { paragités }\end{array}$ & $\begin{array}{r}\text { Pourcent } \\
\text { d'infegtat }\end{array}$ & $\begin{array}{l}\text { sge } \\
\text { ion }\end{array}$ & $\begin{array}{c}\text { Norbire } \\
\mathrm{d}^{+} \text {animaux } \\
\text { paragités }\end{array}$ & $\begin{array}{l}\text { Pourc } \\
\text { d'infe }\end{array}$ & $\begin{array}{l}\text { entage } \\
\text { station }\end{array}$ \\
\hline $\begin{array}{l}\text { Dicrocoelium haspes } \\
\text { Fasciola gientice }\end{array}$ & $\begin{array}{r}8 \\
65\end{array}$ & 56,9 & .100 & $\begin{array}{l}180 \\
112\end{array}$ & $\begin{array}{l}61,3 \\
31,3\end{array}$ & 9.100 \\
\hline $\begin{array}{l}\text { Paremphistomum microbothrium } \\
\text { Celicophoron calicophorum } \\
\text { Gigantocotyle symmeri } \\
\text { Stephanopherymx compectus } \\
\text { Bothriophoron bothriophoron } \\
\text { Total.............................. }\end{array}$ & $\begin{array}{r}41 \\
1 \\
1 \\
0 \\
2 \\
45\end{array}$ & $\begin{array}{r}35,9 \\
0,8 \\
0,8 \\
0 \\
1,7 \\
39,2\end{array}$ & $\begin{array}{l}n \\
" \\
" \\
" \\
"\end{array}$ & $\begin{array}{r}58 \\
3 \\
1 \\
45 \\
46 \\
153\end{array}$ & $\begin{array}{l}19,7 \\
1 \\
0,3 \\
15,3 \\
15,7 \\
52\end{array}$ & $\begin{array}{l}" 1 \\
" \text { " } \\
1 " \\
\text { " } \\
\text { " }\end{array}$ \\
\hline $\begin{array}{l}\text { Carmyerius spatiosus } \\
\text { Carmyerius greberi } \\
\text { Carmyerius papillatus } \\
\text { Carmyerius parvipapillatue } \\
\text { Carmyerius Erefarius } \\
\text { Totel.............................. }\end{array}$ & $\begin{array}{r}15 \\
0 \\
0 \\
1 \\
0 \\
16\end{array}$ & $\begin{array}{l}13 \\
0 \\
0 \\
0,8 \\
0 \\
13,9\end{array}$ & $\begin{array}{l}\text { " } \\
" 1 \\
\text { " }\end{array}$ & $\begin{array}{r}77 \\
2 \\
20 \\
1 \\
4 \\
104\end{array}$ & $\begin{array}{r}26,5 \\
0,6 \\
6,3 \\
0,3 \\
1,2 \\
35,3\end{array}$ & $\begin{array}{l}" 1 \\
" 1 \\
" 1 \\
" 1\end{array}$ \\
\hline $\begin{array}{l}\text { Schistosome bovis } \\
\text { Schistosoma mattheei }\end{array}$ & 61 & 53 & "' & 85 & 28,8 & $"$ \\
\hline $\begin{array}{l}\text { Monjezia expansa } \\
\text { Moniezia benedeni } \\
\text { Thysaniezia ovilla } \\
\text { Total.................. }\end{array}$ & $\begin{array}{r}0 \\
8 \\
5 \\
14\end{array}$ & $\begin{array}{c}0 \\
6,9 \\
5,2 \\
12,1\end{array}$ & " & $\begin{array}{r}1 \\
4 \\
5 \\
10\end{array}$ & $\begin{array}{l}0,3 \\
1,2 \\
1,8 \\
3,3\end{array}$ & $\begin{array}{l}\text { " } \\
\text { " } \\
\text { " }\end{array}$ \\
\hline $\begin{array}{l}\text { Cysticercus bovis } \\
\text { Echinococcus polynorphus }\end{array}$ & $\begin{array}{r}15 \\
2\end{array}$ & $\stackrel{13}{1,7}$ & $"$ & $\begin{array}{r}62 \\
0\end{array}$ & $\begin{array}{r}21 \\
0\end{array}$ & $"$ \\
\hline $\begin{array}{l}\text { Bosicola radjatum } \\
\text { Bunostomum phlobotomum }\end{array}$ & $\begin{array}{r}9 \\
11\end{array}$ & $\begin{array}{l}7,8 \\
9,5\end{array}$ & $\begin{array}{l}n \\
" 1\end{array}$ & $\begin{array}{l}31 \\
16\end{array}$ & $\begin{array}{r}10,4 \\
5,4\end{array}$ & $"$ \\
\hline $\begin{array}{l}\text { Cooperia pectinata } \\
\text { Cooperia punctata } \\
\text { Cooperia sp. } \\
\text { Total................. }\end{array}$ & $\begin{array}{r}13 \\
10 \\
0 \\
23\end{array}$ & $\begin{array}{c}11,4 \\
8,6 \\
0 \\
20\end{array}$ & $\begin{array}{l}" 1 \\
" 1 \\
" 1\end{array}$ & $\begin{array}{r}32 \\
29 \\
2 \\
63\end{array}$ & $\begin{array}{l}10,8 \\
10 \\
0,6 \\
21,4\end{array}$ & $\begin{array}{l}" 1 \\
" 1 \\
" 1\end{array}$ \\
\hline Haemoncus contortus & 0 & 0 & $n$ & 146 & 49,6 & $"$ \\
\hline Haemoncus placei & 0 & 0 & $"$ & 7 & 2,3 & $"$ \\
\hline Artionema labiato-papillosa & 57 & 49,5 & $n$ & 87 & 29,5 & n \\
\hline Onchocerca armilata & 56 & 48,6 & n & 87 & 29,5 & $n$ \\
\hline Onchocerca gutturosa & 67 & 58,2 & $n$ & 127 & 43,2 & n \\
\hline Buckleyruria globulosa & 0 & 0 & & 1 & 0,3 & $\mathbf{m}$ \\
\hline
\end{tabular}


sahéliennes d'élevage dont bequcoup d'animaux sont originaires. Les collections d'eau, les mares permanentes situées le long des «routes du bétail » semblent responsables de cet état de choses (Graber et Thomé, 1964).

Par contre, le bétail de saison des pluies est né ef a grandi dans la région de Maroua. Le parasitisme dont il est afteint est un parasitisme autochtone présentant les caractéristiques signalées plus haut. Sont graves : la Dicrocoeliose, la Distomatose, les Paramphistomoses (Paromphistomidae et Gostrothylacidae), la Schistosomiase, cerfaines Trichostrongyloses ef les Filarioses.

c) La Ladrerie bovine est due à la présence dans les muscles ef dans divers organes de Cysticercus bovis. Elle froppe 21 p. 100 du bétail local, taux bien supérieur à la moyenne du Tchad.

Les localisations changent quelque peu : ainsi les Cysticerques se trouvent, dans 13 p. 100 des cas, dans l'œsophage, ce qui est rarissime au Tchad.

$\begin{array}{lll}\text { Langue : } 29 & \text { Esophage }: 8 \\ \text { Cœur }: 25 & \text { Diaphragme }: 2 \\ \text { Cuisse }: 14 & \text { Foie } & : 1 \\ \text { Psoas }: 9 & \text { Généralisée }: 1\end{array}$

Le nombre de Cysticerques est sujet à d'amples fluctuations :

\begin{tabular}{|c|c|c|c|}
\hline Un & Cysticerque & $: 30$ & nimaux \\
\hline Deux & - & $: 12$ & - \\
\hline Trois & - & $: 9$ & - \\
\hline Quatre & - & $: 5$ & - \\
\hline Cing & - & $: 2$ & - \\
\hline Six & - & $: 1$ & - \\
\hline Sept & - & $: 1$ & - \\
\hline Huit & - & $: 1$ & - \\
\hline Douze & - & $: 1$ & - \\
\hline
\end{tabular}

Par ailleurs, les parasites sont vivants dans les deuxtiers des cas, proportion voisine de celle du Tchad.

d) Dicrocoelium hospes mérite de retenir un peu plus l'attention : sur les 188 bovins purement camerounais atteints, ont été recueillis 9.412 Dicrocoelum, soit en moyenne 50 par animal. 27 d'entre eux (14,3 p. 100) présentaien f plus de 100 Dicrocoelium hospes dans les canaux biliaires, la vésicule et le duodénum :
100 à $150: 13$ animaux

150 à $200: 8$ -

200 à $250: 4-$

$300: 1$ animal

$530: 1 \quad$

II est encore trop tôt de dire à partir de combien d'individus le Trématode est pathogène. Les investigations se poursuivent.

\section{LES ASSOCIATIONS PARASITAIRES}

Sur 140 animaux autopsiés en 1963, il a été possible de dénombrer:

\section{A. - Associations de Trématodes}

67, soit 47,8 p. 100. Elles sont:

10 à deux éléments : 33 (49,2 p. 100).

La plus fréquente est de type $D$. hospes $+F$. gigantica (22 cas sur 33 ) ;

$2^{0}$ à trois éléments : $17(25,2$ p. 100).

Dominent les associations par $D$. hospes $+F$. gigantica $+P$. microbothrium ou $C$. spatiosus ;

$3^{\circ}$ à quatre éléments : 8 (12 p. 100).

dont le modèle est $D$. hospes $+S$. compactus + F. gigantica + C. spatiosus;

40 à cinq éléments : 6 (8,8 p. 100).

Type: D. hospes + P. microbothrium + C. spatiosus $+S$. compactus + B. bothriophoron:

$5^{\circ}$ à six éléments : $3(4,8$ p. 100).

B. - Associations de Trématodes, de Cestodes et de Nématodes (136 des I 40 animaux examinés)

10 à deux éléments: 12 , soit 8,7 p. 100 ;

$2^{\circ}$ à trois éléments : 33 , soił 24,2 p. 100 ,

Types:

F. gigantica $+\mathrm{H}$. contortus + A. labiato-papillosa

D. hospes + S. bovis + O. gutturosa

D. hospes + C. bovis + C. Spatiosus :

$3^{\circ}$ à quatre éléments : 31 , soit 22,7 p. 100.

Types:

D. hospes $+F$. gigantica +0 . armillata +0 . gutturosa 
$D$. hospes $+F$. gigantica $+S$. bovis $+B$. radiotum ;

$4^{\circ}$ à cinq éléments : 27 , soit 19,8 p. 100.

Types :

F. gigantica + C. spatiosus $+S$. compactus + $H$. contortus +0 . gutfurosa

$D$. hospes $+F$. gigantica $+T$. ovilla $+C$. bovis +0. gutturoso

D. hospes $+F$. gigantica $+S$. bovis $+H$. contortus + O. gutturosa ;

50 à six éléments : 24 , soit 17,6 p. 100 ,

Types:

D. hospes + F. gigantica $+S$. bovis $+H$. contortus $+C$. pectinata $+A$. labiato-papillosa

D. hospes $+F$, gigantica + S. compactus + $H$. contortus $+C$. punctata +0 . gutturosa:

60 à sept éléments : 5, soit 3,6 p. 100.

Type :

D. hospes + F. gigantico + S. compoctus $+B$. bothriophoron $+P$. microbothrium $+C$. spotiosus +0 . gutfurosa ;

70 à huit éléments : 2 , soit 1,7 p. 100.

Type : D. hospes $+S$. compoctus $+C$. spotiosus $+B$. bothriophoron $+M$. expansa $+H$. contortus +0 . gutturosa + O. armillata ;

$8^{\circ}$ à neuf éléments : 2 , soit 1,7 p. 100 .

Type :

$D$. hospes $+S$. compactus $+C$. spotiosus $+B$. bothriophoron $+P$. microbothrium $+C$. bovis + $O$. gutturosa $+O$. armillata $+C$. pectinata.

C'est la première fois que des associations à sept, huit et neuf éléments sont observées en Afrıque centrale chez le zébu.

C. - Les associations entre Heiminthes sont donc particulièrement amples et nombreuses chez les animaux de la région de Marova, beaucoup plus en tous cas que chez les anımaux des zones sahéliennes où les associations par 4 et 5 espèces différentes représentent un grand maximum.

En outre, les Helmınthes qui entrent dans la constitution de l'association parasitaire ellemême sont souvent des espèces qui, prises séparément, sont connues pour être passablement pathogènes et dangereuses pour la santé de l'animal (F.' gigantica, C. Spatiosus, S. bovis, Bosicola radiatum...). En association, leurs effets nocifs se conjuguent et c'est ce qui pourrait expliquer - en grande partie - les pertes enregistrées, le mauvais état général et la maigreur des animaux de boucherie du Nord-Cameroun.

\section{CONCLUSIONS}

10 Une enquête menée à l'abattoir de Maroua (Nord-Cameroun) et portant sur 409 animaux de boucherie adultes a permis de mettre en évidence une faune parasitare interne d'une grande richesse et d'une extroordinaire complexité.

Les autopsies ont été effectuées en janvierfévrier 1964, époque où les animaux d'importation originaires des zones sahéliennes de la République du Tchad dominent et en aoûtseptembre 1963-64 où il ne s'agit que de bétail autochtone.

$2^{\circ}$ 'Chez le zébu local, 30 espèces différentes ont été recueillies. Les mieux représentées sont : Dicrocoelium hospes (61,3 p. 100), Fasciolo gigantica (31,3 p. 100), divers Paramphistomidae de la panse (52 p. 100$)$, cing Carmyerius ( 35,3 p. 100$)$, Schistosoma bovis (28,8 p. 100), Cysticercus bovis (21 p. 100), de nombreux Trichostrongylidae (Haemoncus contortus, 52 p. 100 ; Cooperia pectinota et Cooperia punctata, 21 p. 100) et des Filaires (Artionema labiato-popillosa, 29,5 p. 100 ; Onchocerca armillato, 29,5 p. 100 ; Onchocerca gutturosa, 43,2 p. 100).

Le parasitisme par Esophagostomes et Bunostomes semble assez modéré. L'incidence du Téniasis est négligeable.

30 Les résultats de l'enquête indiquent également que les zébus originaires de la République du Tchad et abattus à Maroua sont beaucoup plus atteints que dans leur pays d'origine (zones sahéliennes) : les méthodes africaines traditionnelles de commercialisation du bétail et les longs déplacements qu'il faut dans le sens Nord-Sud sont sans doute à l'origine de cet état de choses.

4a La comparaison entre les taux moyens d'infestation du bétail au Tchad, en R. C. A. (Bouar) et à Maroua montre que, dans cette région, le parasitısme occupe une position intermédiaire. 
Plus on descend vers les zones humides (Isohyètes 750 à $1.500 \mathrm{~mm}$ ), plus les infestations parasitaires sont fortes et nombreuses.

$5^{\circ}$ Au Nord-Cameroun, les associations entre Helminthes sont une règle quasi constante. Elles sont graves parce qu'elles mettent en jeu sou- vent six, huit et même neuf espèces différentes dont l'action pathogène individuelle est tenue pour sérieuse - tout au moins pour certaines d'entre elles.

Les associations compliquent singulièrement la prophylaxie à mettre en œuvre.

\section{SUMMARY}

Helminths of adult zebus in the Maroua area (Nort-Cameroons)

A survey made at the Maroua abattoir in North Cameroons on 409 beef cattle animals has given evidence in the native stock of 30 different helminths species. The most common are: Dicrocoelium hospes $(61,3 \mathrm{p} .100)$, Fasciola gigantico (31,3 p. 100) several Omentum paramphistomidoe (52 p. 100), five cormyerius $(35,3$ p. 100), Schistosoma bovis (28,8 p. 100), Cysticercus bovis (21 p. 100) numerous strongyllidae (Hoemoncus contortus 52 p. 100, Cooperia pectinata, Cooperio punctata 21 p. 100) and filaricie (Artionéma labiato papilloso 29,5 p. 100) ; Onchocerca armillata 29,5 p. 100 , Onchocerca gutfurosa 43,2 p. 100). Parasitic condition due to Oesophagostomum radiotum, Bunostomum phlebotornum and the great intestinal cestodes seems rather moderate.

These worms are associated together in most cases, and these associations are harmful because they put together often up to 9 species among which some are highiy pathogenic,

The authors compare the incidence of parasitism in the adult : North Cameroons zebu and the globol incidence in the zebus of Chad and Centrafrican Republic (Bouar). They point out al a noticeable increase of the infestation ratio when animals from the sahel zones (rains 300 to $500 \mathrm{~m} / \mathrm{m}$ ) go down to moister zones (rains $800-900 \mathrm{~mm}$ ) to be slaughtered.

\section{RESUMEN}

\section{Helmíntos de los cebús adultos de largión de Marua (Norte-Cameron)}

Una encuesta hecha en el matadero de Marua (Norte Cameron) concerniendo a 409 animales de carniceria permitıó demostrar en el ganado autóctono 30 diferentes especies de heimíntos entre los cuales se notan :

Dicrocaelium hospes $(61,3$ por 100$)$.

Fascrolo gigantica (31,3 par 100).

Algunos Paramfistomidae de la barriga (52 par 100).

Cinco Carmyerius $(35,3$ por 100$)$.

Schistosoma bovis $(28,8$ por 100$)$.

Cysticercus bovis (21 por 100).

Numerosos strongylidae (Hoemoncus contortus 52 por 100 ; Cooperia pectinata y cooperio punctato 21 por 100).

Y filarios Artionema labiato-papillosa (29.5 por 100).

Onchocerca armillato $(29,5$ por 100$)$.

Onchocerca gutturoso $(43,2$ por 100).

El parasitismo con Oesophagostomum radiatum, Bunuosiomum phieotombny los grandes céstados del intestino delgado parece poco importante.

Estos heimíntos estan ligados entre ellos en casi todos los casos y las asocia ciones son dañinas por que mezclan a menudo hasta nueve especies cuyas algunas son muy patogenas.

Los autores comparan el parasitismo del cebu adulto del norte-cameron con el global de los cebus del Chad y de là Republica centroafricana (Bouar) Notan un neto crecimiento del termino medio de infestatión de los ánimales de las zonas sahelianas (lluvias $300-500 \mathrm{~mm}$ ) cuando bojan hacia regiones más húme dos (l!uvias $800-900 \mathrm{~mm}$ ) para la matanza. 


\section{BIBLIOGRAPHIE}

1. ABDEL MALEK (E.). - Occurrence of Onchocerca armillato Railliet et Henry 1909 in Sudanese cattle Bos indicus. J. Porosit. (1958), 44, 4 (sect. 2), 30-1.

2. CHODNIK (K. S.). - Aortic Onchocerciasis due to $O$. armillato in cattle in Ghana, with special reference to the morphology of the parasite. Ann. Trcp. Med. Porasit. (1957), 51, 2, 216-224.

3. CHODNIK (K. S.). - Histopathology of aortic lesions in cattle infected with $O$. armillata. Ann. Trop. Med. Parasit. (1958), 52, 2, 145-8.

4. DOLLFUS (P. H.) et DEVIGNE (R.). - Fourmis responsables de la propagation de la « petlte Douve » Dicrocoelium lanceolatum (Rudolphi, 1803), du mouton en Lorraine. Observations sur la larve métacercaire. $C . R$. Acad. Sc. Paris (1965), 269, 6, gr. 12, $1758-$ 1760.

5. EUZEBY (J.). - Diagnostic expérimental des Helminthoses animales. Vigot, Paris (1958), 367 PP.

6. FAIN (A.), HERRIN (V.) et THIENPONT (D.). - Filariose des bovidés au Ruanda-Urundi. III. Etude parasitologique. Filaires des genres Sefaria et Onchocerca ef microfilaires sanguines et dermiques. Ann. Soc. Belge Med. Trop. (1955), 35, 5, 555-583.

7. GRABER (M.). - Le parasitisme des animaux domestiques en République centrafricaine. Données préliminaires. Monographie Laboratoire Farcha-Service Elevage. R. C. A. (1961), 65 PP.

8. GRABER (M.), DOUTRE (M.), FINELLE (P.), KERAVEC (J.), DUCROZ (G.) et MOKOTAINGAR (P.). - Les Helminthes de quelques Artiodactyles squvages appartenant auX familles des Bovidés et des Suidés. Ces Mammifères, en République du Tchad et en R.C.A. sont-lls des réservoirs de parasites pour les animaux domestiques qui vivent à leur contact. Rev. Elev. Med. Vet. Poys Trop. (1964), 17, 3, 377-420.

9. GRABER (M.) et THOME (M.). - La Cysticercose bovine en République du Tchad. Quelques réflexions sur la situation présente, l'étiologie, le diagnostic, l'immunité ef le traitement de cette zoonose. Rev. Elev. Med. Vet. Pays Trop. (1964), 17, 3, 441-466.
10. GRABER (M.) et OUMATIE (O.). - Existence en Afrique équatoriale d'un important foyer de Dicrocoeliose bovine et ovine à Dicrocoelium hospes (Looss, 1907). Rev. Elev. Med. Vet. Pays Trop. (1964), 17, 3, 523533.

11. GRABER (M.). - Parasitoses internes. Rap. Ann. Ministère agriculture et Production animale Tchad, fasc. VII (1964), 89-127.

12. GRETILLAT (S.). - Maintien du genre Bothriophoron Stiles ef Goldberger, 1910 et valeur de l'espèce Paramphistomum bothriophoron (Braun, 1892) Fischoeder, 1901 (Trematoda, Poramphitomidae) parasite du reticulum du zébu malgache. Ann. Porosit. Hum. Comp. (1958), 33, 3, 240-253.

13. GRETILLAT (S.). - Amphistomes (Trematodes) des ruminants domestiques de la République du Tchad. Description d'un Gasfrothylacidae nouvedu Carmyerius graberin. Sp. Ann. Porasit. Hum. Comp. (1960), 35, 4, 509-527.

14. GRETILLAT (S.). - Carmyerius papillatus N. Sp. ef Carmyerius parvipapillatus N. Sp. (Trematoda, Gastrothylacidae) parasites des réservoirs gastriques de l'antilope Kobus defassa Rupp. Ann. Parasit. Hum. Comp. (1962), 37, 1/2, 121-139.

15. GRETILLAT (S.). - Sur quelques Poramphistomoidea (Trematoda) d'une collection du Musée royal de I'Afrique centrale. Rev. Zool. Bot. Afr. (1964), LXIX, 3/4, 351-367.

16. GRETILLAT (S.) et PICART (P.). - - Premières observations sur les lésions provoquées chez les ruminants infestés massivement par Shistosoma curassoni. Rev. Elev. Vet. Pays Trop. (1964), 17, 3, 433-440.

17. HERIN (V.), THIENPONT (D.) et FAIN (A.) - Filarioses des Bovidés au Ruanda-Urundi. I. Etude clinique. Ann. Soc. Belge. Med. Trop. (1955), 35, 5, 502-521.

18. NASMARK (K. E.). - A revision of the Trematode family Poromphistomidae. Inaug. Disserf. Zool. Bidrag. Uppsolo (1937), 16, 301-566, 104 fig., 13 pl.

19. SPASSKI (A. A.). - Anoplocephalate tapeworms of domestic and wild animals. Acod. Sc. U.R. S. S. (1951), Moscou, 783 Pp. 291 fig. 